\title{
Novel structural approaches concerning HPV proteins: Insight into targeted therapies for cervical cancer (Review)
}

\author{
KALLIOPI I. PAPPA ${ }^{1,2}$, GEORGIA KONTOSTATHI ${ }^{2,3}$, VASILIKI LYGIROU ${ }^{2,3}$, \\ JEROME ZOIDAKIS $^{3}$ and NICHOLAS P. ANAGNOU ${ }^{2,4}$
}

\begin{abstract}
${ }^{1}$ First Department of Obstetrics and Gynecology, University of Athens School of Medicine, Alexandra Hospital, Athens 11528;
${ }^{2}$ Laboratory of Cell and Gene Therapy, and ${ }^{3}$ Biotechnology Division, Biomedical Research Foundation of the Academy of Athens, Athens 11527; ${ }^{4}$ Laboratory of Biology, University of Athens School of Medicine, Athens 11527, Greece
\end{abstract}

Received August 7, 2017; Accepted October 16, 2017

DOI: $10.3892 /$ or.2018.6257

\begin{abstract}
Cervical cancer incidence is tightly linked to HPV infection, and particularly virus types 16 and 18 cause the majority of cases presenting with pre-cancerous stages of cervical intraepithelial neoplasia (CIN). Structural and functional information concerning HPV proteins can offer novel insight into the mechanism(s) of cancer progression in the cervical epithelium. Recently, novel structural determinants of the interactions of viral proteins with their targets in keratinocytes have been elucidated. These exciting findings open the way for the development of targeted anti-oncogenic therapies, and may eventually allow the introduction of novel approaches for a rational cervical cancer treatment.
\end{abstract}

\section{Contents}

1. Introduction: HPV and cervical cancer

2. Structural information on the key HPV proteins

3. Potential for targeted therapy

4. Perspectives

\section{Introduction: HPV and cervical cancer}

Cervical cancer is attributed to human papillomaviruses (HPVs). Depending on their oncogenic potential, HPVs are divided into low-risk types that predominantly cause benign warts and high-risk types associated with malignant disease (1). There are $\sim 15$ high-risk mucosal HPV types of which the most important ones are HPV16 and HPV18, which

Correspondence to: Professor Kalliopi I. Pappa, First Department of Obstetrics and Gynecology, 80 Vasilisis Sofias Avenue, University of Athens School of Medicine, Athens 11528, Greece

E-mail: kalliopi.pappa20@gmail.com

Key words: HPV oncoproteins, cervical cancer, structure, function, targeted therapy together account for more than $70 \%$ of cervical carcinomas in women (2). The genome of HPV16 and HPV18 contains a long control region (LCR) and eight genes implicated in the virus life cycle. Binding sites for transcription factors and the viral E1 and E2 proteins are located in the LCR. The expression of viral mRNAs is controlled by two promoters, early promoter (PE) and late promoter (PL) in HPV16 and HPV18 (3).

Viral entry occurs through a minor abrasion of the cervical epithelium, which allows HPV virions to infect the basal strata. Upon entry, during the establishment phase, the virus is maintained as an episome at $\sim 50-100$ copies in the infected cells, inducing early intraepithelial lesions such as CIN1. The first viral genes expressed are the oncogenes E6 and E7 in the lower strata cells; this expression results in cell cycle deregulation and subsequent proliferation, while in the upper layers, genome amplification occurs through the expression of other proteins such as E1, E2, E4 and E5, with these cells residing in the $S$ or $G_{2}$ phase of the cell cycle. Exit from the cell cycle in these cells occurs through a viral copy number increase up to many thousands of copies/cell. Furthermore, the ensuing expression of the viral L1 and L2 proteins in E4-positive cells, allows the packaging and generation of novel infectious viral particles. In the second or the maintenance phase of the viral life cycle, the virus is integrated into the host genome, leading to a gradual progression to cervical cancer via the CIN2 and CIN3 stages. Finally, in the vegetative or productive phase, amplification of the viral DNA occurs in high copy numbers in the differentiated cells, resulting in its packaging in viral capsids. In established cervical cancer, the integration of the viral genome results in the abnormal overexpression of E6 and E7, and the loss of expression of full-length E1, E2, E4 and E5 as well as L1 and L2 capsid proteins (3-5). The identification and assessment of all the proteins expressed in the HPV genome, has opened the way for detailed studies of their molecular function, in view of the limited therapeutic modalities for cervical cancer during the last 20 years (6). Currently, major efforts focusing on immunotherapy have not yet yielded a significant breakthrough (7).

Thus, the aim of the present review is to present the available information on the structural approaches concerning the various key HPV proteins and discuss how this novel insight 
could lead to targeted therapeutic approaches for cervical cancer.

\section{Structural information on the key HPV proteins}

L1 and L2 proteins. The capsid of HPV is composed of two proteins, L1 and L2. L1 exhibits significant differences among HPV types, whereas the L2 sequence is more conserved. L1 protein is the major protein of a virus-like particle (VLP), while the L2 protein constitutes the minor protein. The identification of a heparin-binding C-terminus domain of the L1 of HPV11 type, suggests that the initial attachment of the virion to host cells is mediated by the exposure of the basal membrane of keratinocytes and binding of L1 to heparin sulfate proteoglycans (8). The mechanism of viral entry includes the binding of VLPs to the $\alpha 6$ integrin subunit and in turn, these complexes utilize $\beta 1$ or $\beta 4$ integrin as co-receptors that facilitate HPV binding and entry into epithelial cells (9). The capsid of the HPV16 VLP has been studied at different stages of maturation by cryo-electron microscopy and image analysis. The size of VLPs varies during maturation. The VLP structures are stabilized by inter-L1 disulfide bonds and their cross-linking increases towards more mature forms (10). It has been suggested that C161, C229 and C379, which are highly conserved in different HPV types, are actually involved in the early phases of virion assembly, by creating transient disulfide bonds, as was shown mainly by the mutational analysis of the above cysteines (11). The VLP surface displays loops projecting to the exterior surface, containing epitopes that are recognized by the immune system. Five loops have been identified (BC, DE, EF, FG and HI) which contain epitopes that generate specific antibody responses. Three different structural states of L1 HPV16, an L1 monomer, an L1 pentamer and a VLP consisting of 12 pentamers arranged in an icosahedral structure, exhibit different immunogenicity. The icosahedral VLP is the most effective in eliciting an immune response (12). In another study, crystal structures of L1 pentamers of high-risk HPV16, HPV18, HPV35 and low-risk HPV11 were resolved. The $\beta$-sheets of all HPV types are identical but important conformational changes occur on their pentamer surfaces, including a few angstrom $(\AA)$ shift of the surface loop or some substitutions of the surface loop residues. The structural differences of the pentamers are possibly responsible for the binding specificity of monoclonal antibodies that are raised against the VLP of the corresponding HPV type (13). Furthermore, the properties of the L1 monomer of cutaneous and mucosal HPVs, i.e. HPV5 and HPV16, respectively, have been compared and their 3D-structures were analyzed in order to visualize the number of exposed charged amino acids of the L1 protein. The number of negatively charged amino acids was higher in HPV5 than in HPV16. L1 HPV5 is negatively charged at physiological $\mathrm{pH}$ 7.40, compared to positively charged HPV16 L1 at the same $\mathrm{pH}$. In the same study, the uptake of HPV5 and HPV16 was determined using pseudoviruses in two cancer cell lines, the cervical C33A and the cutaneous HaCaT. The negatively charged heparin did not inhibit the uptake of HPV5 in C33A cells and slightly inhibited its uptake in $\mathrm{HaCaT}$ cells. On the contrary, it inhibited the HPV16 uptake in HaCaT cells and completely blocked its uptake in C33A cells. These differences between HPV5 and HPV16 could reflect differences of the HPV tropism between cutaneous and mucosal HPV types (14).

The L2 protein in HPVs represents the minor protein of the capsid (15). It promotes the transportation of the virion into the nucleus of the host cell by interacting with host dynein following endosomal entry (16). HPV16 L2 contains 462 amino acids and can enter the nucleus via $K a p \alpha_{2} \beta_{1}$, $\operatorname{Kap} \beta_{2}$ and $K a p \beta_{3}$ receptors (17). The structures of different L2 proteins and their interaction with L1 have been studied in silico. Different Alpha-HPV sequences were evaluated and it was shown that five loops of L1 (BC, DE, EF, FG and HI), are the most variable regions among the Alpha-HPVs, whereas specific regions of L2 interacting with L1, are evolutionarily conserved. Furthermore, specific regions of L1 that interact with $\mathrm{L} 2$ regions are also conserved. A predicted 3D model indicated the interaction between L1 and L2, via the direct involvement of DE and FG L1 loops and L2 proline rich areas (18). In order to study in vitro the interaction of L1 and L2, HPV16 capsids or pseudoviruses were produced in 293T cells. Capsids were separated by sodium dodecyl sulfatepolyacrylamide gel electrophoresis (SDS-PAGE) and the evaluation of L1 and L2 band intensities yielded a L1:L2 ratio of 5:1. Computerized reconstructions of L1 and L2 capsids revealed pentavalent capsomers, whereas cryo-electron microscopy showed an icosahedrally ordered L2-specific density beneath the axial lumen of each L1 capsomer. Using bimolecular fluorescence and 'split GFP' technology, L2 proteins were shown to be located in close proximity in the virion structure (15). A proposed model for the entry of HPV16 in keratinocytes, involves binding via the L1 major capsid protein to heparin sulfate proteoglycans (HSPGs) on the epithelial cell surface or the basement membrane. Growth factor receptors form complexes with HSPGs and HPV16, thus inducing conformational changes of the virion. Subsequently, isomerization of the virion by cyclophilin B and proprotein convertase occurs, leading to $\mathrm{L} 2$ minor capsid protein cleavage and the increase of L2 $\mathrm{N}$-terminus exposure. The binding of HPV16 to $\alpha 6$ integrins promotes further signaling cascades inside the cell, by binding to L2-specific receptors, such as Annexin A2 heterotetramers, leading to clathrin-, caveolin-, lipid raft-, flotillin-, cholesterol- and dynamin-independent endocytosis of HPV16 (19). The role of Annexin A2 heterotetramer has been studied in terms of viral infection, since the S100A10 subunit of the heterotetramer based on immunoprecipitation studies, interacts with HPV16 L2, facilitating viral entry in knockdown experiments (20). In addition, the entry of HPV51 was found to be mediated by the transport protein particle complex subunit 8 (TRAPPC8) following HPV binding to HSPGs. The transport protein particle (TRAPP) complex regulates multiple membrane trafficking pathways and TRAPPC8 is one of its subunits expressed in human tissues. As proposed, TRAPPC8-dependent endocytosis transports the capsid into the cell. After conformational rearrangements of the capsid, the $\mathrm{L} 2 \mathrm{~N}$-terminus is exposed and interacts with TRAPPC8, blocking the function of TRAPPC8. As a result, the endosome is degraded and the viral genome is released from the trans-Golgi network (21). In conclusion, L1 and L2 proteins are responsible for the virion assembly and are involved in crucial interactions with cellular macromolecules that facilitate viral entry to keratinocytes. 
E1 protein. The E1 protein acts as a DNA-dependent adenosine triphosphatase (ATPase) and DNA helicase; these enzymatic activities are required for the initiation of viral DNA replication. E1 protein forms a complex with E2 protein that binds to the origin of replication of the viral DNA, which contains binding sites for both proteins. In particular, binding of an E1 dimer to an E2 dimer occurs, forming a complex that binds to the origin of replication with high specificity. The E2 dimer is displaced by a second E1 dimer, in an ATP-dependent manner, resulting in E1 tetramer formation. The next step is the binding of two E1 monomers to each half of the origin of replication, which results in the formation of two E1 trimers. The final result is the formation of two hexamers to the viral origin, each hexamer located to each half of the origin of replication, that act as a helicase, unwinding the viral DNA and eventually recruiting the host DNA polymerase to initiate replication $(22,23)$. A highly conserved $\mathrm{N}$-terminal region of E1 among different HPV types was found by deletion analysis, to be necessary for efficient replication of viral DNA. The above region forms an amphipathic $\alpha$-helix (AH) and a specific peptide within binds to the $\mathrm{p} 62$ protein subunit of the human homolog of transcription factor TFHII. Mutational analysis of the three conserved hydrophobic residues in the E1 $\mathrm{AH}$, revealed a $50 \%$ reduction of the ability of $\mathrm{E} 1$ to support transient replication of DNA in C33A cells (24). Human p80 is an important co-partner of all mucosal HPV E1 proteins. P80 binds to the N-terminal 40 amino acids of HPV31 E1. Amino acid substitutions in this region, cause $70 \%$ reduction of viral DNA replication. The structure of the $\mathrm{N}$-terminal 40-amino acid-long peptide originating from HPV31 E1, was solved by NMR. Overexpression of this 40-amino acid-long p80-binding peptide, derived from HPV31 E1, led to the inhibition of viral DNA replication preventing the recruitment of endogenous p80 to the origin of replication. Thus, the E1-p80 interaction represents a promising new target for antiviral therapy (22). In another study, the structure of the DNA-binding domain of E1 HPV18 was resolved by crystallography and was compared to the respective structure of DNA-binding domain from E1 of the bovine papillomavirus type 1 (BPV1). The DNA-binding loop in HPV18 E1 displays a disordered pattern of organization in contrast to the respective loop in E1 BPV1. The $\alpha 3$ helix of the HPV18 E1 DNA-binding domain (DBD) is a residue shorter than the analogous BPV1 E1-DBD $\alpha 3$ helix. The $\alpha 3$ helix is the dimerization helix and the process of dimerization occurs through its hydrophobic residues of each monomer, as confirmed by mutational analysis and ChiP assays (25). In conclusion, the structure of E1 HPV protein allows binding to specific origins of replication of viral DNA and its distinct domains display an ATP-dependent DNA helicase activity that facilitates viral DNA replication.

E2 protein. Regulatory protein E2 is involved in the initiation of viral DNA replication through the interaction with E1. E2 acts as a transcription factor by binding to the E2 response element (E2RE), which is the major E2-dependent enhancer in the LCR, as shown in the BPV1 genome (23). The structure of E2 protein reveals its discrete functions, as far as cell transformation is concerned. There are three basic domains in E2 which include the DBD at the C-terminus, the flexible hinge in the middle and the transactivation domain (TAD) at the N-terminus of the protein (26). The structures of the DBDs of different high- and low-risk HPVs were resolved by crystallography. Moreover, the interaction of the DBD of E2 proteins with the LCR of the viral DNA was evaluated. It is interesting to note that the structure of the DBD from the HPV6 E2 protein, shows higher structural homology to the equivalent DBD from the HPV18 E2 and BPV1 E2 proteins than the DBD from HPV16 E2. The most common DNA binding site for HPV16 and HPV6 E2 DBDs is the sequence 5'-AACCGN ${ }_{4}$ CGGTT-3', where $\mathrm{N}_{4}$ represents a 4-bp central spacer sequence. This central spacer contains sequences rich in AT that promote DNA bending and increases protein binding (27). The interaction of E2 with E1 was studied by the use of small molecules that bind to the N-terminal TAD of HPV11 E2 and inhibit its interaction with E1. These inhibitors contain an indandione system spirofused onto a substituted tetrahydrofuran ring, and abolish DNA replication. The study of the tertiary structure of the low-risk HPV11 E2 TAD is interesting, as it resembles the corresponding domain of HPV16. The crystal structures of the HPV11 E2 TAD with the above inhibitors, were resolved by crystallography and the specific amino acids that form the inhibitor binding pocket have been identified. These amino acids are located in a three-helix formation and amino acid substitutions in this area alter the binding pocket, by changing the packing of these three helices (28). DNA binding properties of HPV6 E2 DBD and a corresponding mutated DBD lacking two C-terminal leucine residues that form part of the hydrophobic core of the protein, were compared by employing NMR. It was proven that the hydrophobic core and loop regions that guide the DNA binding helices are highly flexible in the mutant. This increased flexibility resulted in non-specific DNA binding and loss of E2 protein function (29). In conclusion, the current data indicate that E2 protein facilitates initiation of viral replication via its interactions with the E1 protein.

E4 protein. E4 protein of HPV16 is expressed as an E1^E4 transcript of 92 amino acids (30). The process of alternative splicing generates the $\mathrm{E} 1^{\wedge} \mathrm{E} 4$ protein, in which the initiation codon and the first few amino acids originate from the E1 open reading frame (31). E4 is detectable in the upper layers of the epithelium, expressed at the late phases of the HPV life cycle (23). The N-terminus of HPV16 E2 protein was proven to interact with the $\mathrm{E} 1^{\wedge} \mathrm{E} 4$ protein through direct binding, leading to the stabilization of E2 and translocation from the nucleus to the cytoplasm (32). A mammalian expression system was used to demonstrate that HPV16 E1^E4 arrests HeLa $\left(\mathrm{HPV} 18^{+}\right)$and SiHa $\left(\mathrm{HPV} 16^{+}\right)$cervical epithelial cells in the $G_{2}$ phase. Mutagenesis analysis revealed an important region rich in prolines in $\mathrm{HPV} 16 \mathrm{E} 1 \wedge \mathrm{E} 4$ responsible for the $\mathrm{G}_{2}$ arrest, which contains a putative nuclear localization signal, a cyclin-binding motif, and a single cyclin-dependent kinase phosphorylation site. The arrest domain of HPV16 E1^E4 is similar to the corresponding $\mathrm{E} 1^{\wedge} \mathrm{E} 4$ of HPV11, a low-risk mucosal type, which also causes $\mathrm{G}_{2}$ arrest. However, E1^E4 from HPV1, a low-risk cutaneous type which does not mediate $\mathrm{G}_{2}$ arrest, exhibits significant differences at the sequence level when its arrest domain was aligned with E1^E4 from HPV16 and HPV11. This structural difference is responsible for the inability of HPV1 E1^ $\mathrm{E} 4$ to induce arrest at the $\mathrm{G}_{2}$ phase (33). 
Mutational analysis of HPV1 E4 and HPV16 E4, revealed that the C-terminal domain of HPV16 E1^ ${ }^{\wedge} 4$ is crucially involved in the disruption of the keratin filament network (34). HPV16 $\mathrm{E} 1^{\wedge} \mathrm{E} 4$ protein associates with the keratin intermediate filament network as it was shown by immunofluorescence microscopy. This conserved function amongst the HPV Alpha-group E1^ ${ }^{\wedge} 4$ proteins, has been demonstrated by amino acid sequence alignment. Hyperphosphorylated and ubiquitinated E1^E4-keratin structures have been documented, resulting in the impairment of the proteasome and causing the disruption of the keratin network (35). In conclusion, E4 protein is expressed in the upper layers of the cervical epithelium and its biological functions involve cell cycle arrest and disruption of keratin filaments.

E5 protein. HPV16 E5 is a transmembrane protein of 83 amino acids, localized in the Golgi apparatus (GA) and the endoplasmic reticulum (ER). The protein folds in three hydrophobic domains which contain a high percentage (37\%) of $\alpha$-helical structure (36). HPV16 E5 regulates growth signaling pathways as it activates the epidermal growth factor receptor (EGFR) and the downstream Ras-Raf-MAP kinase pathway or the PI3K-Akt pathway that lead to altered cell proliferation, angiogenesis and inhibition of apoptosis (37). The HPV E5 protein has been shown to contribute to immune evasion as it interacts with MHC/HLA class I, at the GA, where it accumulates. As a result, HPV16 E5 prevents transport of MHC-I to the cell surface and retains the complex in the GA, attenuating the recognition of a viral epitope by natural killer (NK) cells $(37,38)$. The biological effect of E5 is mainly mediated by its interaction with the HLA-I heavy chain. Furthermore, the first hydrophobic domain is absolutely required for the downregulation of surface HLA-I and interaction with the heavy chain (38). In another study, the $\mathrm{pH}$ status of different intracellular compartments was tested upon the presence of the E5 protein. The transduction of human keratinocytes with an E5 vector increased endosomal $\mathrm{pH}$ from 5.9 to 6.9, but it did not affect the normal trans-Golgi $\mathrm{pH}$ of 6.3. Endosomal location of E5 increases $\mathrm{pH}$ and presumably enhances EGFR signaling (39). The expression pattern of HPV16 and HPV18 E5 was evaluated by creating specific ${ }^{3} \mathrm{H}$-labeled riboprobes targeting the corresponding mRNAs. The E5 mRNA and protein of these HPV types were found in anogenital LSIL but not in cervical tumors, suggesting a role of E5 in the early stages of HPV infection (40). A proposed mechanism for the interference of HPV16 E5 in apoptotic pathways has been described. HPV16 E5 is responsible for an antiapoptotic effect by impairing tumor necrosis factor-related apoptosis-inducing ligand (TRAIL) and Fas ligand (FasL)mediated apoptosis in two different ways. Firstly, HPV16 E5 downregulates the levels of Fas receptor in the cell surface, thus preventing its interaction to its corresponding ligand. Secondly, HPV16 E5 prevents the formation of the deathinducing signalling complex (DISC) which is induced by TRAIL (41). One important target of HPV16 E5 was proven to be interferon $\beta-1$ (IFN- $\beta 1$ ), its expression known to be facilitated by the increased interferon regulatory factor 1 (IRF-1) protein accumulation in human keratinocytes. A proposed model of action during the episomal infection of HPV, assumes that HPV16 E5 stimulates IFN- $\beta 1$ and IRF-1 expression that lead to episomal clearance. Following this, only transcripts of integrated viral genes remain, E5 expression is abolished and the IFN-IRF-1 mediated response is shut down. Meanwhile, high levels of E6 and E7 oncogenes suppress the signaling pathway of IFN (42). In a comparative study of BPV1 E5 and HPV16 E5, the transforming properties of the two different E5 proteins were evaluated. Epitope-tagged BPV1 E5 and HPV16 E5 proteins were expressed at the same level in fibroblast cell lines. However, only BPV1 E5 but not HPV16 E5 could activate growth factor receptors, phosphoinositide 3-kinase or c-Src. In fibroblast cell line NIH 3T3 co-cultured with vectors expressing either BPV1 E5 or HPV16 E5, only cells that expressed BPV1 E5 formed visible foci, underscoring the limited proliferating capacity of cells expressing HPV16 E5 (43). E5 has also been shown to be responsible for cell transformation when acting with other oncoproteins. For example, with the contribution of E6 it results in the formation of koilocytes, as was demonstrated in human cervical cells in vitro. Koilocytes, which are squamous epithelial cells that contain an acentric, hyperchromatic nucleus that is displaced by a large perinuclear halo, constitute a morphological marker of HPV infection (44). A proposed model for the formation of koilocytes includes E5-induced translocation of calpactin I to the perinuclear region which promotes perinuclear membrane fusion (45). A novel partner for HPV31 E5 seems to be the proteolipid protein A4 which is localized to the ER with E5. This interaction facilitates the differentiation of keratinocytes, as was confirmed in organotypic raft cultures, with these cells expressing high levels of A4 (46).

The role of HPV16 E5 was also demonstrated in the $\mathrm{HaCaT}$ human keratinocyte model. E5-expressing cells formed a highly abnormal epithelium, where many cells exhibited characteristics of cells derived from the basal membrane, such as production of matrix metalloproteases (MMPs). It was documented by mutational analysis, that the first hydrophobic domain of E5 is required for invasion. The four C-terminal amino acids induce the production of MMP9, presumably by increasing the levels of transcription factor AP-1 that binds to the MMP9 promoter (47). In conclusion, E5 protein is responsible for immune system evasion of the HPV, induction of apoptosis, cell transformation and episomal clearance.

E6 protein. HPV E6 proteins are quite small in size of $\sim 150$ amino acids, and include two zinc-binding domains E6N and E6C (48). The structure of the E6 homodimer was characterized by NMR where it was shown that E6 dimer disruption through mutations at the E6N domain, enhances E6 solubility and subsequent reduction of p53 degradation potential. The E6N and E6C domain structures were resolved by NMR studies demonstrating that E6 specifically dimerizes through its $\mathrm{N}$-terminal domain. It has been proposed that each E6 molecule of the dimer binds to one molecule of ubiquitin ligase E6-associated protein (E6AP) and to one molecule of p53 in order to promote the polyubiquitination of p53 by E6AP, resulting in p53 degradation (Fig. 1). Dimers of E6 are formed in high-risk mucosal HPV16 and HPV18 types, as well as in low-risk mucosal type HPV11, whereas E6 from cutaneous strains HPV5 and BPV1 is monomeric. However, only the dimeric E6 protein from oncogenic types HPV16 and HPV18 has the ability to polyubiquitinate p53. The structural basis for 

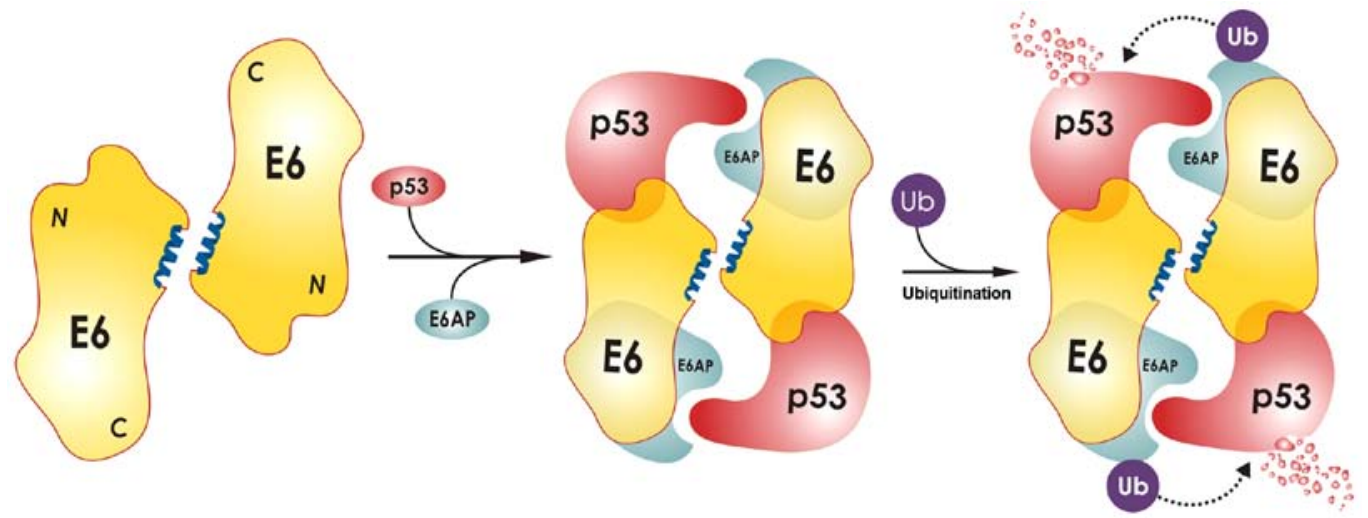

Figure 1. Important functions of the E6 oncoprotein. Dimerization model of E6 through its N-terminal domain and particularly the $\alpha 2$ helices (shown in blue). In high-risk HPV types, p53 degradation occurs by the dimerization model of E6 through its N-terminusl domain. Each E6 molecule of the dimer binds to one molecule of ubiquitin ligase E6AP and to one molecule of $\mathrm{p} 53$ in order to promote the polyubiquitination of p53 by the E6/E6AP complex. Polyubiquitination of p53 leads to its degradation, cell cycle deregulation and subsequent abnormal cell proliferation. N, N-terminus, C, C-terminus.

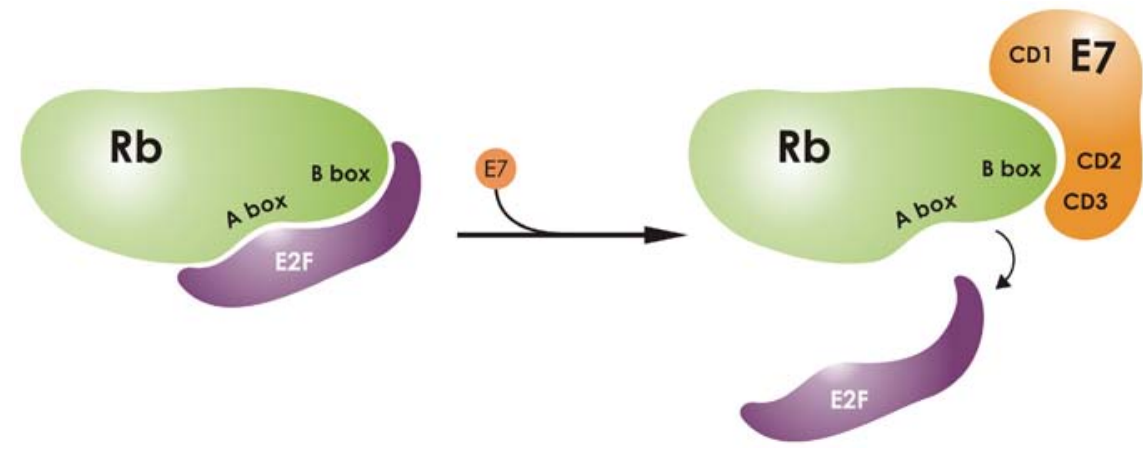

Figure 2. Interaction of the E7 oncoprotein with Rb. Initially, E2F binds to Rb, via the A and B Box. E7 protein contains three conserved regions CD1, CD2 and CD3 which contains the Cys-X-X-Cys motifs. Following the interaction of E7 with Rb, CD2 binds to the B box of Rb through its LXCXE motif. The flexibility of $\mathrm{E} 7$ enables the binding of the $\mathrm{CD} 3$ region to the Rb B box. Through these interactions, the Rb-E2F complex is disrupted, thus leading to an early and abnormal cell progression into the $\mathrm{S}$ phase of the cell cycle.

the inability of the HPV11 E6 dimer to target p53 for degradation is currently under investigation (49). E6 protein from high-risk HPV types also binds to proteins containing PDZ domains and targets them for degradation. The interaction of the complex of the human homologue of the Drosophila discs large tumor suppressor protein (hDlg) bound to HPV18 E6 was analyzed by NMR (50). The structure of the second zincbinding domain (51Z2) of the high-risk HPV51 E6 protein was also resolved by NMR spectroscopy. Specifically, circular dichroism spectroscopy revealed an increased flexibility of the above area of E6, indicating that this may be the structural feature of E6 oncoproteins. Another study investigated the E6AP binding site of E6 protein in HPV16. The conclusion was that an inhibitory ligand can bind to E6 protein at its E6AP binding pocket, restore p53 expression and induce apoptosis in HPV16 positive cells. This important finding opens new perspectives for drug development against HPV (51). In a subsequent study, more details were provided concerning the specific interactions of E6, E6AP and p53. In particular, the crystal structure of the ternary complex containing the proteins of interest HPV16 E6, the LxxLL motif of E6AP and the core domain of p53, was determined. The LxxLL motif is a short leucine (L)-rich LxxLL consensus sequence within the ubiquitin ligase domain of E6AP that binds to E6. The formation of the ternary complex results in ubiquitin-mediated p53 degradation. Mutations of E6 residues that establish interactions with p53 abolish complex formation and p53 degradation (52). An equally important function of the E6 protein is the upregulation of hTERT telomerase expression; only high-risk HPV E6 proteins bind to the promoter of hTERT telomerase and induce its expression. Another interesting function of E6 proteins both in high- and in low-risk HPV types, is their ability to interact with caspase 8 , through the DED region of caspase. The result is the localization of caspase 8 to the nucleus, as shown by binding assays and immunofluorescence experiments. The nuclear localization of caspase 8 is required for the facilitation of the viral cycle and the proliferation of cells that express E6, presumably through regulation of apoptotic signals (53). In conclusion, E6 interacts with p53 and proteins with PDZ domains leading to their degradation, while increases hTERT expression, and thus promoting cell cycle deregulation and subsequent malignant transformation.

E7 protein. The structure of the HPV18 E7 protein contains three discrete domains, CD1, CD2 and CD3. These domains are also called CR1, CR2 and CR3, respectively. E7 protein has one $\mathrm{Zn}^{2+}$ ion which coordinates to four cysteines at the C-terminal domain through the two Cys-X-X-Cys motifs 
located in the CD3 domain, thus forming a hydrophobic core. $\mathrm{Zn}^{2+}$ plays a crucial role in the maintenance of structural integrity of HPV18 E7 and is absolutely required for proper folding and thermal stability of the protein. The N-terminal domain is flexible and binds to the $\mathrm{pRb}$ protein via its LXCXE motif, which is located in the CD2 region (54). In a crystallography study of the CD3 domain of E7, a zinc binding domain was characterized and was implicated in the dissociation of $\mathrm{pRb}$ from E2F transcription factors and the early cell progression into the $\mathrm{S}$ phase of the cell cycle. By performing mutational analysis, two specific binding sites were discovered in the CD3 region. The first is necessary for $\mathrm{pRb}$ binding, whereas the other is necessary for $\mathrm{E} 2 \mathrm{~F}$ binding (Fig. 2). Interaction of pRb with E7 is necessary to disrupt the pRb-E2F complex (55). A crystallography analysis of the pocket domain of the retinoblastoma $(\mathrm{Rb})$ protein, revealed its interaction with a nine-residue E7 peptide containing the LxCxE motif. The crystallography study revealed the presence of two boxes in the Rb pocket, the A- and the B-box. The E7 LxCxE was shown to bind to an extended peptide onto a conserved groove of the B Box (Fig. 2). A recent study links the conformational status of HPV16 E7 to functional redox roles, as the protein contains seven highly conserved cysteines. These are modified under oxidative stress that characterizes HPV-transformed cells. There are two distinct redox centers, cysteine 24 located in the E7N domain, which contains the Rb binding site, and also cysteines 59/68 located in the E7C domain, which contains the $\mathrm{Zn}^{2+}$ binding site. Under oxidative stress, glutathionylation of cysteine 24 abolishes Rb-binding. Binding of $\mathrm{Zn}^{2+}$ to cysteine residues 59 and 68 , mediates structural rearrangements leading to disulfide bridging of the above cysteines (56). Moreover, it was demonstrated that HPV8 E7 is imported in the nucleus of HeLa cells, following transfection with enhanced green fluorescent protein 8E7 (EGFP-8E7), and that the actual role of cysteine residues seems to be significant. Mutations in cysteine residues involved in zinc coordination alter the nuclear localization of EGFP-8E7 (57). The entire structure of HPV16 E7 protein was recently resolved by NMR studies, which revealed that the E7 heterogeneity and dynamics, containing a flexible N-terminus and a more structured C-terminus, may be responsible for the oncogenic potential of E7 and its interactions with other important biomolecules (58). These interactions of E7 with its protein targets result in uncontrolled cell division and subsequent malignant transformation.

\section{Potential for targeted therapy}

Delineation of the interactions of HPV oncoproteins E6 and E7 with their multiple cellular targets, can provide the impetus for an eventual targeted drug therapy for cervical cancer. Specifically, an in silico study suggested that plant compounds could be used to treat cervical cancer since they can block the binding of HPV18 E6 to p53, and thus prevent its degradation (59). Moreover, competitive antagonist ligands, i.e. benzopyranone derivatives, that disrupt the E6-E6AP interaction, were developed in order to inhibit the formation of the complex containing E6, E6AP and p53 (Fig. 1). These promising anticancer agents were used in binding and functional assays (60). Another promising agent for cervical cancer therapy is a peptide that targets the E6AP binding pocket of E6 from HPV16 and prevents p53 degradation (61). As far as therapy via targeting of $\mathrm{E} 7$ is concerned, an in vitro characterization and screening of compounds that inhibited the ability of HPV-E7 to disrupt pRB/E2F complexes, has been recently conducted. Thiadiazolidinedione-based molecules exhibited the highest affinity for E7 and effectively prevented its binding to $\mathrm{pRB}$. In addition, some of these compounds were proven to induce apoptosis in HeLa and SiHa cervical cell lines. It is important to note that one molecule was tested in mice injected with the TC-1 lung cancer-derived cell line and was found to significantly reduce the tumor volume without any adverse effects (62).

The elucidation of the structural features of the E6 oncoprotein responsible for establishing interactions with protein targets, has provided the basis for potential pharmacological interventions. In particular, the PDZ-binding motif (PBM) of E6, is involved in the formation of complexes with proteins containing PDZ domains and leads to their degradation (50). An in silico analysis revealed four compounds that could disrupt the interaction of the E6 PBM with the PDZ domain. These compounds target specifically the PDZ domain via the interaction with Gly463 and Phe464 (63). However, the in silico prediction has to be validated experimentally and thus, the molecular therapy of targeting the PBM, is currently at an early stage of development. As previously demonstrated by Zanier et al (51), eventual molecular therapy for cervical cancer can also be developed by disrupting the interactions of the E6AP binding site and the E6 protein of HPV16, via an inhibitory ligand that binds to E6 protein. Furthermore, in vitro experiments by the same group, indicate that p53 expression can be restored by treating HPV16-positive cells with selected compounds, leading to apoptosis (49). In a related study, the interaction of the HPV16 E6 oncoptotein with regulators of apoptosis caspase 8 and E6AP was targeted, in order to reveal putative drugs. Screening of compound libraries for such putative inhibitors was performed and two compounds, myricetin and spinacine, were found to inhibit the binding of E6 to caspase 8 and E6AP. These two molecules had a significant cytotoxic effect specifically for the HPV16-positive cervical cancer cell line SiHa (64). These compounds exhibit $\mathrm{IC}_{50}$ value in the $\mu \mathrm{M}$ range and offer a proof of principle for therapeutic targeting of HPV E6. Finally, an additional study was based on screening a library of 88,000 compounds for inhibitors of the E6-E6AP interaction. Seven inhibitors were identified by cell-free ELISA assays and two compounds exhibited significant cytotoxic activity towards $\mathrm{SiHa}$ and $\mathrm{HeLa}$ (HPV18-positive) cells. It is important to note that treatment of these cervical cancer cell lines with the two compounds restored $\mathrm{p} 53$ expression and the $\mathrm{IC}_{50}$ values reached the $\mathrm{nM}$ range (65). This seminal study opens the way for testing the efficacy of such promising compounds for the treatment of cervical cancer.

Therefore, besides the functional studies on informative cervical cell lines in vitro $(66,67)$, further efforts are also needed employing appropriate in vivo models of cervical cancer in order to fully evaluate the effectiveness of these novel targeted therapies. 


\section{Perspectives}

Structural studies have revealed important differences between HPV proteins from high- and low-risk HPV types. The structural differences are responsible for different binding partners and offer insight into the mechanisms of HPV-mediated carcinogenesis. In particular, E6 and E7 proteins from highrisk HPV types are responsible for inducing uncontrolled cell proliferation. Only high-risk E6 binds p53 and targets it for ubiquitination, whereas high-risk E7 binds the non-phosphorylated form of the $\mathrm{Rb}$ protein and facilitates the cell to bypass the $\mathrm{G}_{1} / \mathrm{S}$ checkpoint.

The structural information on HPV proteins can be used to facilitate research for the development of novel therapeutic targets and new types of vaccines for the prevention and eventual therapy of cervical cancer. In conclusion, insight from the structural studies of HPV proteins has contributed to the elucidation of molecular mechanisms associated with cervical carcinogenesis and may open the way for the development of novel therapeutic approaches.

\section{Acknowledgements}

The present review was co-funded by the European Union [European Social Fund and Greek National Fund (ESF)], through the Program THALIS, under the Operational Program Education and Lifelong Learning of the National Strategic Reference Framework (NSRF), project no. 383418, grant no. 70-3-11830 to K.I.P.

\section{References}

1. Hoory T, Monie A, Gravitt P and Wu TC: Molecular epidemiology of human papillomavirus. J Formos Med Assoc 107: 198-217, 2008

2. Muñoz N, Bosch FX, Castellsagué X, Díaz M, de Sanjose S, Hammouda D, Shah KV and Meijer CJ: Against which human papillomavirus types shall we vaccinate and screen? The international perspective. Int J Cancer 111: 278-285, 2004.

3. Doorbar J, Quint W, Banks L, Bravo IG, Stoler M, Broker TR and Stanley MA: The biology and life-cycle of human papillomaviruses. Vaccine 30 (Suppl 5): F55-F70, 2012.

4. Stanley MA: Epithelial cell responses to infection with human papillomavirus. Clin Microbiol Rev 25: 215-222, 2012.

5. McBride AA: Replication and partitioning of papillomavirus genomes. Adv Virus Res 72: 155-205, 2008.

6. Dueñas-González A and Campbell S: Global strategies for the treatment of early-stage and advanced cervical cancer. Curr Opin Obstet Gynecol 28: 11-17, 2016.

7. Schiffman M, Doorbar J, Wentzensen N, de Sanjosé S, Fakhry C, Monk BJ, Stanley MA and Franceschi S: Carcinogenic human papillomavirus infection. Nat Rev Dis Primers 2: 16086, 2016.

8. Joyce JG, Tung JS, Przysiecki CT, Cook JC, Lehman ED, Sands JA, Jansen KU and Keller PM: The L1 major capsid protein of human papillomavirus type 11 recombinant virus-like particles interacts with heparin and cell-surface glycosaminoglycans on human keratinocytes. J Biol Chem 274: 5810-5822, 1999.

9. Evander M, Frazer IH, Payne E, Qi YM, Hengst K and McMillan NA: Identification of the alpha6 integrin as a candidate receptor for papillomaviruses. J Virol 71: 2449-2456, 1997.

10. Cardone G, Moyer AL, Cheng N, Thompson CD, Dvoretzky I, Lowy DR, Schiller JT, Steven AC, Buck CB and Trus BL: Maturation of the human papillomavirus 16 capsid. MBio 5: e01104-e01114, 2014.

11. Ryndock EJ, Conway MJ, Alam S, Gul S, Murad S, Christensen ND and Meyers C: Roles for human papillomavirus type 1611 cysteine residues 161, 229, and 379 in genome encapsidation and capsid stability. PLoS One 9: e99488, 2014.
12. Joshi H, Cheluvaraja S, Somogyi E, Brown DR and Ortoleva P: A molecular dynamics study of loop fluctuation in human papillomavirus type 16 virus-like particles: A possible indicator of immunogenicity. Vaccine 29: 9423-9430, 2011.

13. Bishop B, Dasgupta J, Klein M, Garcea RL, Christensen ND, Zhao R and Chen XS: Crystal structures of four types of human papillomavirus L1 capsid proteins: Understanding the specificity of neutralizing monoclonal antibodies. J Biol Chem 282: 31803-31811, 2007.

14. Mistry N, Wibom C and Evander M: Cutaneous and mucosal human papillomaviruses differ in net surface charge, potential impact on tropism. Virol J 5: 118, 2008.

15. Buck CB, Cheng N, Thompson CD, Lowy DR, Steven AC, Schiller JT and Trus BL: Arrangement of L2 within the papillomavirus capsid. J Virol 82: 5190-5197, 2008.

16. Schneider MA, Spoden GA, Florin L and Lambert C: Identification of the dynein light chains required for human papillomavirus infection. Cell Microbiol 13: 32-46, 2011.

17. Darshan MS, Lucchi J, Harding E and Moroianu J: The 12 minor capsid protein of human papillomavirus type 16 interacts with a network of nuclear import receptors. J Virol 78: 12179-12188, 2004.

18. Lowe J, Panda D, Rose S, Jensen T, Hughes WA, Tso FY and Angeletti PC: Evolutionary and structural analyses of alphapapillomavirus capsid proteins yields novel insights into L2 structure and interaction with L1. Virol J 5: 150, 2008.

19. Raff AB, Woodham AW, Raff LM, Skeate JG, Yan L, Da Silva DM, Schelhaas M and Kast WM: The evolving field of human papillomavirus receptor research: A review of binding and entry. J Virol 87: 6062-6072, 2013.

20. Woodham AW, Da Silva DM, Skeate JG, Raff AB, Ambroso MR, Brand HE, Isas JM, Langen R and Kast WM: The S100A10 subunit of the annexin A2 heterotetramer facilitates L2-mediated human papillomavirus infection. PLoS One 7: e43519, 2012.

21. Ishii Y, Nakahara T, Kataoka M, Kusumoto-Matsuo R, Mori S, Takeuchi T and Kukimoto I: Identification of TRAPPC8 as a host factor required for human papillomavirus cell entry. PLoS One 8: e80297, 2013.

22. Lehoux M, Fradet-Turcotte A, Lussier-Price M, Omichinski JG and Archambault J: Inhibition of human papillomavirus DNA replication by an E1-derived p80/UAF1-binding peptide. J Virol 86: 3486-3500, 2012.

23. Peter M: Howley DRL: Papillomaviruses. Journal II: 2300-2354, 2007.

24. Morin G, Fradet-Turcotte A, Di Lello P, Bergeron-Labrecque F, Omichinski JG and Archambault J: A conserved amphipathic helix in the N-terminal regulatory region of the papillomavirus E1 helicase is required for efficient viral DNA replication. J Virol 85: 5287-5300, 2011.

25. Auster AS and Joshua-Tor L: The DNA-binding domain of human papillomavirus type $18 \mathrm{E} 1$. Crystal structure, dimerization, and DNA binding. J Biol Chem 279: 3733-3742, 2004.

26. Bellanger S, Tan CL, Xue YZ, Teissier S and Thierry F: Tumor suppressor or oncogene? A critical role of the human papillomavirus (HPV) E2 protein in cervical cancer progression. Am J Cancer Res 1: 373-389, 2011.

27. Dell G, Wilkinson KW, Tranter R, Parish J, Leo Brady R and Gaston K: Comparison of the structure and DNA-binding properties of the E2 proteins from an oncogenic and a non-oncogenic human papillomavirus. J Mol Biol 334: 979-991, 2003.

28. Wang Y, Coulombe R, Cameron DR, Thauvette L, Massariol MJ, Amon LM, Fink D, Titolo S, Welchner E, Yoakim C, et al: Crystal structure of the E2 transactivation domain of human papillomavirus type 11 bound to a protein interaction inhibitor. J Biol Chem 279: 6976-6985, 2004.

29. Brown C, Campos-León K, Strickland M, Williams C Fairweather V, Brady RL, Crump MP and Gaston K: Protein flexibility directs DNA recognition by the papillomavirus E2 proteins. Nucleic Acids Res 39: 2969-2980, 2011.

30. Nakahara T, Peh WL, Doorbar J, Lee D and Lambert PF: Human papillomavirus type 16 E1circumflexE4 contributes to multiple facets of the papillomavirus life cycle. J Virol 79: 13150-13165, 2005.

31. Doorbar J: The E4 protein; structure, function and patterns of expression. Virology 445: 80-98, 2013.

32. Davy C, McIntosh P, Jackson DJ, Sorathia R, Miell M, Wang Q, Khan J, Soneji Y and Doorbar J: A novel interaction between the human papillomavirus type $16 \mathrm{E} 2$ and $\mathrm{E} 1^{\wedge} \mathrm{E} 4$ proteins leads to stabilization of E2. Virology 394: 266-275, 2009. 
33. Davy CE, Jackson DJ, Wang Q, Raj K, Masterson PJ, Fenner NF Southern S, Cuthill S, Millar JB and Doorbar J: Identification of $\mathrm{a}_{2}$ arrest domain in the $\mathrm{E} 1$ wedge $\mathrm{E} 4$ protein of human papillomavirus type 16. J Virol 76: 9806-9818, 2002.

34. Roberts S, Ashmole I, Rookes SM and Gallimore PH: Mutational analysis of the human papillomavirus type $16 \mathrm{E} 1 \wedge \mathrm{E} 4$ protein shows that the $\mathrm{C}$ terminus is dispensable for keratin cytoskeleton association but is involved in inducing disruption of the keratin filaments. J Virol 71: 3554-3562, 1997.

35. McIntosh PB, Laskey P, Sullivan K, Davy C, Wang Q, Jackson DJ, Griffin HM and Doorbar J: E1^E4-mediated keratin phosphorylation and ubiquitylation: A mechanism for keratin depletion in HPV16-infected epithelium. J Cell Sci 123: 2810-2822, 2010

36. Yang DH, Wildeman AG and Sharom FJ: Overexpression, purification, and structural analysis of the hydrophobic E5 protein from human papillomavirus type 16. Protein Expr Purif 30: 1-10, 2003.

37. Venuti A, Paolini F, Nasir L, Corteggio A, Roperto S, Campo MS and Borzacchiello G: Papillomavirus E5: The smallest oncoprotein with many functions. Mol Cancer 10: 140, 2011.

38. Ashrafi GH, Haghshenas M, Marchetti B and Campo MS E5 protein of human papillomavirus 16 downregulates HLA class I and interacts with the heavy chain via its first hydrophobic domain. Int J Cancer 119: 2105-2112, 2006.

39. Disbrow GL, Hanover JA and Schlegel R: Endoplasmic reticulum-localized human papillomavirus type $16 \mathrm{E} 5$ protein alters endosomal $\mathrm{pH}$ but not trans-Golgi $\mathrm{pH}$. J Virol 79: 5839-5846, 2005.

40. Stoler MH, Rhodes CR, Whitbeck A, Wolinsky SM, Chow LT and Broker TR: Human papillomavirus type 16 and 18 gene expression in cervical neoplasias. Hum Pathol 23: 117-128, 1992.

41. Kabsch $\mathrm{K}$ and Alonso A: The human papillomavirus type 16 E5 protein impairs TRAIL- and FasL-mediated apoptosis in $\mathrm{HaCaT}$ cells by different mechanisms. J Virol 76: 12162-12172, 2002.

42. Muto V, Stellacci E, Lamberti AG, Perrotti E, Carrabba A, Matera G, Sgarbanti M, Battistini A, Liberto MC and Focà A: Human papillomavirus type 16 E5 protein induces expression of beta interferon through interferon regulatory factor 1 in human keratinocytes. J Virol 85: 5070-5080, 2011.

43. Suprynowicz FA, Disbrow GL, Simic V and Schlegel R: Are transforming properties of the bovine papillomavirus E5 protein shared by E5 from high-risk human papillomavirus type 16 ? Virology 332: 102-113, 2005.

44. Krawczyk E, Suprynowicz FA, Liu X, Dai Y, Hartmann DP, Hanover J and Schlegel R: Koilocytosis: A cooperative interaction between the human papillomavirus E5 and E6 oncoproteins. Am J Pathol 173: 682-688, 2008.

45. Krawczyk E, Suprynowicz FA, Hebert JD, Kamonjoh CM and Schlegel R: The human papillomavirus type $16 \mathrm{E} 5$ oncoprotein translocates calpactin I to the perinuclear region. J Virol 85: 10968-10975, 2011.

46. Kotnik Halavaty K, Regan J, Mehta K and Laimins L: Human papillomavirus E5 oncoproteins bind the A4 endoplasmic reticulum protein to regulate proliferative ability upon differentiation. Virology 452-453: 223-230, 2014.

47. Barbaresi S, Cortese MS, Quinn J, Ashrafi GH, Graham SV and Campo MS: Effects of human papillomavirus type 16 E5 deletion mutants on epithelial morphology: Functional characterization of each transmembrane domain. J Gen Virol 91: 521-530, 2010

48. Nominé Y, Charbonnier S, Ristriani T, Stier G, Masson M, Cavusoglu N, Van Dorsselaer A, Weiss E, Kieffer B and Travé G: Domain substructure of HPV E6 oncoprotein: Biophysical characterization of the E6 C-terminal DNA-binding domain. Biochemistry 42: 4909-4917, 2003.

49. Zanier K, ould M'hamed ould Sidi A, Boulade-Ladame C, Rybin V, Chappelle A, Atkinson A, Kieffer B and Travé G: Solution structure analysis of the HPV16 E6 oncoprotein reveals a self-association mechanism required for E6-mediated degradation of p53. Structure 20: 604-617, 2012.

50. Liu Y, Henry GD, Hegde RS and Baleja JD: Solution structure of the hDlg/SAP97 PDZ2 domain and its mechanism of interaction with HPV-18 papillomavirus E6 protein. Biochemistry 46: 10864-10874, 2007.
51. Zanier K, Stutz C, Kintscher S, Reinz E, Sehr P, Bulkescher J, Hoppe-Seyler K, Travé G and Hoppe-Seyler F: The E6AP binding pocket of the HPV16 E6 oncoprotein provides a docking site for a small inhibitory peptide unrelated to E6AP, indicating druggability of E6. PLoS One 9: e112514, 2014.

52. Martinez-Zapien D, Ruiz FX, Poirson J, Mitschler A, Ramirez J, Forster A, Cousido-Siah A, Masson M, Vande Pol S, Podjarny A, et al: Structure of the E6/E6AP/p53 complex required for HPV-mediated degradation of p53. Nature 529: 541-545, 2016.

53. Manzo-Merino J, Massimi P, Lizano M and Banks L: The human papillomavirus (HPV) E6 oncoproteins promotes nuclear localization of active caspase 8. Virology 450-451: 146-152, 2014.

54. Liu S, Tian Y, Greenaway FT and Sun MZ: A C-terminal hydrophobic, solvent-protected core and a flexible N-terminus are potentially required for human papillomavirus $18 \mathrm{E} 7$ protein functionality. Biochimie 92: 901-908, 2010.

55. Liu X, Clements A, Zhao K and Marmorstein R: Structure of the human Papillomavirus E7 oncoprotein and its mechanism for inactivation of the retinoblastoma tumor suppressor. J Biol Chem 281: 578-586, 2006.

56. Chemes LB, Camporeale G, Sánchez IE, de Prat-Gay G and Alonso LG: Cysteine-rich positions outside the structural zinc motif of human papillomavirus E7 provide conformational modulation and suggest functional redox roles. Biochemistry 53 : 1680-1696, 2014.

57. Onder Z and Moroianu J: Nuclear import of cutaneous beta genus HPV8 E7 oncoprotein is mediated by hydrophobic interactions between its zinc-binding domain and FG nucleoporins. Virology 449: 150-162, 2014.

58. Calçada EO, Felli IC, Hošek T and Pierattelli R: The heterogeneous structural behavior of E7 from HPV16 revealed by NMR spectroscopy. ChemBioChem 14: 1876-1882, 2013.

59. Kumar S, Jena L, Sahoo M, Kakde M, Daf S and Varma AK: In silico docking to explicate interface between plant-originated inhibitors and E6 oncogenic protein of highly threatening human papillomavirus 18. Genomics Inform 13: 60-67, 2015.

60. Rietz A, Petrov DP, Bartolowits M, DeSmet M, Davisson VJ and Androphy EJ: Molecular probing of the HPV-16 E6 protein alpha helix binding groove with small molecule inhibitors. PLoS One 11: e0149845, 2016

61. Stutz C, Reinz E, Honegger A, Bulkescher J, Schweizer J, Zanier K, Travé G, Lohrey C, Hoppe-Seyler K and HoppeSeyler F: Intracellular analysis of the interaction between the human papillomavirus type 16 E6 oncoprotein and inhibitory peptides. PLoS One 10: e0132339, 2015.

62. Fera D, Schultz DC, Hodawadekar S, Reichman M, Donover PS, Melvin J, Troutman S, Kissil JL, Huryn DM and Marmorstein R: Identification and characterization of small molecule antagonists of $\mathrm{pRb}$ inactivation by viral oncoproteins. Chem Biol 19: 518-528, 2012.

63. Tian YS, Kawashita N, Arai Y, Okamoto K and Takagi T: Pharmacophore modeling and molecular docking studies of potential inhibitors to E6 PBM-PDZ from human papilloma virus (HPV). Bioinformation 11: 401-406, 2015.

64. Yuan CH, Filippova M, Krstenansky JL and DuerksenHughes PJ: Flavonol and imidazole derivatives block HPV16 E6 activities and reactivate apoptotic pathways in $\mathrm{HPV}^{+}$cells. Cell Death Dis 7: 2060, 2016.

65. Malecka KA, Fera D, Schultz DC, Hodawadekar S, Reichman M, Donover PS, Murphy ME and Marmorstein R: Identification and characterization of small molecule human papillomavirus E6 inhibitors. ACS Chem Biol 9: 1603-1612, 2014.

66. Kontostathi G, Zoidakis J, Makridakis M, Lygirou V, Mermelekas G, Papadopoulos T, Vougas K, Vlamis-Gardikas A, Drakakis P, Loutradis D, et al: Cervical cancer cell line secretome analysis highlights the role of transforming growth factor-betainduced protein ig-h3, peroxiredoxin-2 and NRF2 on cervical cancer carcinogenesis. BioMed Res Int 2017: 4180703, 2017.

67. Pappa KI, Lygirou V, Kontostathi G, Zoidakis J, Makridakis M, Vougas K, Daskalakis G, Polyzos A and Anagnou NP: Proteomic analysis of normal and cancer cervical cell lines reveals deregulation of cytoskeleton-associated proteins. Cancer Genomics Proteomics 14: 253-266, 2017. 